\title{
Outpatient bilateral supracostal tubeless percutaneous nephrolithotomy for staghorn calculi
}

\author{
Andrea Kokorovic; James W.L. Wilson, MD, FRCSC; Darren Beiko, MD, MBA, FRCSC
}

Department of Urology, Queen's University, Kingston General Hospital, Kingston, ON

Cite as: Can Urol Assoc J 2014;8(3-4):e273-5. http://dx.doi.org/10.5489/cuaj.1691

Published online April 14, 2014.

\section{Abstract}

Percutaneous nephrolithotomy (PCNL) is the surgical procedure of choice to treat staghorn calculi. Most centres perform PCNL as the traditional inpatient procedure. However, outpatient PCNL has been successfully attempted and represents a feasible method of reducing hospital costs. We report the case of a 35-year-old female who underwent outpatient simultaneous PCNL for bilateral renal staghorn calculi. The patient was discharged in stable condition less than 3 hours following the procedure with minimal discomfort. To the best of our knowledge, this case report is the first to describe a successfully completed outpatient bilateral supracostal tubeless PCNL for staghorn calculi.

\section{Introduction}

Percutaneous nephrolithotomy (PCNL) has been accepted as the gold standard treatment for large and complex renal calculi. ${ }^{1}$ Since its emergence in $1976,{ }^{2}$ numerous modifications have been made to the procedure. The traditional method, which continues to be widely used, involves placing an indwelling nephrostomy tube. ${ }^{2}$ The development of a tubeless PCNL procedure has resulted in decreased postoperative morbidity and lower length of hospital admission. Multiple studies to date have demonstrated that this is a safe and effective method of stone burden clearance in select patients. ${ }^{1,3-7}$ Recently, tubeless PCNL, on an outpatient basis, has been reported..$^{8,9}$ This case report is the first to describe a case of outpatient bilateral tubeless PCNL in a patient with bilateral large staghorn calculi.

\section{Case report}

A 35-year-old healthy female presented with a 2-year history of recurrent urinary tract infection (UTI). Infections resolved temporarily with antibiotics; however, symptom recurrence ensued following a completed course of therapy, while prophylactic therapy was unsuccessful. Urine culture identified staphylococcus saprophyticus and coagulase negative staphylococcus species. Renal ultrasound and computed tomography (CT) scan with CT-KUB (kidneys-ureters-bladder) scout film revealed mild hydronephrosis and bilateral staghorn calculi (Fig. 1). A branched calculus measuring $3.6 \times 3.2 \times 3.0 \mathrm{~cm}$ filled the renal pelvis, lower pole and interpolar calyces on the right and a branched calculus measuring $4.0 \times 3.0 \times 1.6 \mathrm{~cm}$ filled the renal pelvis, lower pole calyces, interpolar calyces and upper pole infundibulum on the left. Despite the significant stone burden, the patient experienced minimal discomfort and there was no history of septicemia.

Treatment strategies for the large stone burden were discussed, and bilateral PCNL was proposed. The risks of the procedure were discussed, including bleeding and infection, and informed consent was obtained. The patient was prescribed oral levofloxacin $500 \mathrm{mg}$ daily for 7 days immediately prior to PCNL.

The patient was brought to the operating room in stable condition. Cefazolin $(1 \mathrm{~g})$ and gentamicin $(80 \mathrm{mg})$ were administered intravenously for prophylaxis, followed by induction of general anesthesia. Flexible cystoscopy was performed with the patient in the prone position. The right and left ureteral orifices were catheterized with a 5-Fr openend ureteral catheter. An 18-Fr Foley catheter was introduced into the bladder and placed to straight drainage.

Attention was first turned to the right kidney. Right retrograde pyelography outlined the collecting system and stones; above the 11th rib, a supracostal approach was used to puncture the tip of the upper pole posterior calyx under fluoroscopy. A skin incision was made and the tract was dilated using a balloon dilator and the nephroscope sheath was inserted. Rigid nephroscopy revealed a large stone burden in the renal pelvis that extended into interpolar and lower pole calyces. The staghorn calculus was fragmented and removed using a combination of ultrasonic lithotripsy, stone 


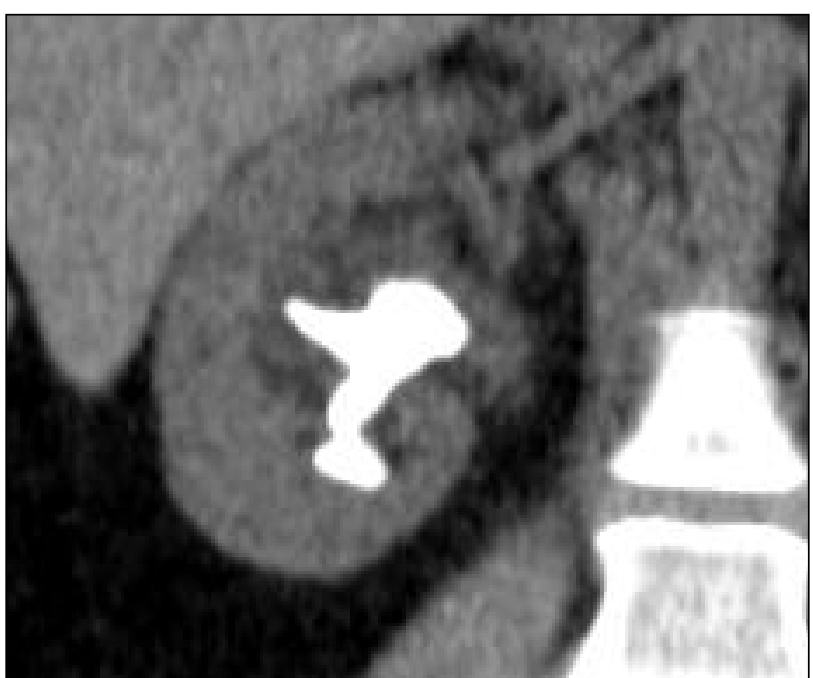

Fig. 1a. Coronal computed tomography image showing right staghorn calculus.

graspers and a nitinol stone basket. Antegrade pyelography did not demonstrate any residual stones or filling defects, and full nephroscopy, using both the rigid and flexible nephroscope, did not reveal any residual fragments. A 6-Fr, $24-\mathrm{cm}$ ureteral stent was placed in an antegrade fashion. The skin was closed with a horizontal mattress 4-0 monocryl suture and steri-strips. There was negligible bleeding during right PCNL.

Attention was then turned to the left kidney. Left retrograde pyelography demonstrated a greater stone burden, with filling defects identified in the renal pelvis, lower pole calyx, interpolar calyces and the infundibulum of the upper pole calyx. Once again, supracostal percutaneous access was achieved above the 11 th rib at the tip of the upper pole posterior calyx. Tract dilation and nephroscopy ensued, and a combination of ultrasonic lithotripsy, grasper extraction and basket extraction was used to clear the large stone burden. Antegrade pyelography and full nephroscopy did not reveal any filling defects or residual stones whatsoever. A 6-Fr, 24-cm ureteral stent was placed in an antegrade fashion. The skin was closed with a horizontal mattress 4-0 monocryl suture. Again, bleeding was negligible during left PCNL. Total operative time, including positioning and prone cystoscopy, was 203 minutes.

The patient was transferred to the post-anesthetic care unit, where she was given fentanyl $(25 \mathrm{mcg})$ intravenously and experienced minimal discomfort. Her Foley catheter was removed 2 hours postoperatively, and she was voiding well prior to discharge. Vital signs remained stable, and postoperative hemoglobin was $133 \mathrm{~g} / \mathrm{L}$, with a preoperative value of $136 \mathrm{~g} / \mathrm{L}$. Chest x-ray showed no pneumothorax bilaterally. The patient was discharged in stable condition with minimal postoperative pain 2 hours and 52 minutes following the procedure, with a prescription for 1 to 2 Percocet

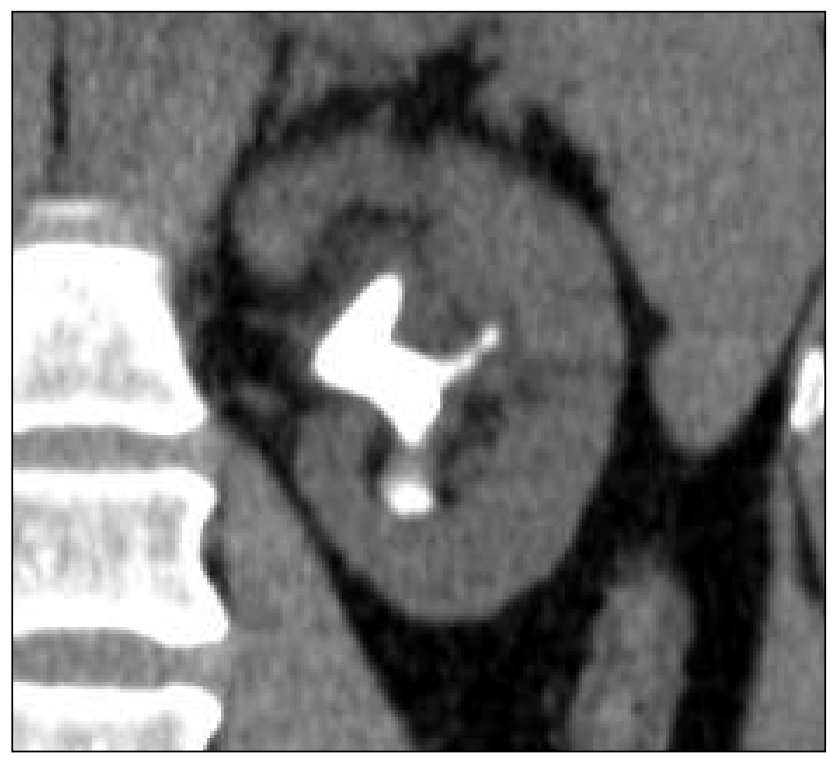

Fig. 1b. Coronal computed tomography image showing left staghorn calculus.

(Endo Pharmaceuticals, oxycodone hydrochloride and acetaminophen) tablets orally every 4 hours as required and oral levofloxacin $500 \mathrm{mg}$ daily for 1 week. At the 6-week follow-up appointment, the patient was doing well with no complaints or complications. At this time, a follow-up KUB x-ray did not show any obvious residual fragments and both ureteral stents were removed cystoscopically at the same time. Stone analysis revealed calcium carbonate/ phosphate with magnesium ammonium phosphate calculi. The 3-month follow-up ultrasound suggested a 7-mm right lower pole calculus. However, the patient remains asymptomatic and is under surveillance to assess for possible stone growth and UTI in the future.

\section{Discussion}

PCNL has gained wide acceptance as the procedure of choice for large and complex renal calculi. ${ }^{1}$ The original procedure, introduced in 1976, was developed using nephrostomy tubes to secure the tract and to impede postoperative hemorrhage. ${ }^{2}$ However, the high rates of morbidity in the form of postoperative discomfort, and the prolonged duration of hospitalization with this method have inspired the development of numerous variations to the procedure.

The most widely used of these variations is tubeless PCNL, whereby a ureteral stent replaces the traditional nephrostomy tube. ${ }^{3}$ Numerous studies have supported the use of the tubeless method as a viable alternative to standard PCNL. ${ }^{1,4-7}$ The advantages of tubeless PCNL over the standard procedure include reduced postoperative analgesic requirements, as well as reduced duration of hospitalization without an increase the rate of complications. ${ }^{1,4-7}$ 
Surgical advancements have enabled the use of tubeless PCNL in complex cases, including pediatric, geriatric and bilateral simultaneous renal calculi. ${ }^{10-12}$ Furthermore, ambulatory (outpatient) PCNL has been successfully attempted, and represents a safe and feasible surgical option for carefully selected patients., ${ }^{910}$ Tubeless PCNL represents an economically favourable approach to treat renal calculi. Reduced length of hospitalization is a major cost-saving advantage of the procedure, allowing for reallocation of limited healthcare resources. Outpatient tubeless PCNL improves the cost-effectiveness of the procedure by further decreasing the length of hospital stay and avoiding the potential costs associated with nosocomial infections. Unfortunately, despite the advantages offered by the tubeless approach, many centres continue to use the standard method. Perhaps the limitations of tubeless PCNL, such as lack of access to perform second-look nephroscopy, accounts for the lack of widespread adoption of tubeless PCNL in many countries.

\section{Conclusion}

This case report is, to our knowledge, the first to describe an outpatient bilateral tubeless PCNL procedure, particularly using a supracostal approach for bilateral staghorn calculi. The patient did not experience any intraoperative or postoperative complications, and was discharged with minimal discomfort. Importantly, she was cleared of any significant stone burden at 3 months, as confirmed by a follow-up KUB and ultrasound. Outpatient tubeless bilateral PCNL enhances the aforementioned economic benefits of the bilateral tubeless procedure and offers an achievable method of reducing hospital costs. Although discharging patients mere hours following bilateral PCNL may be viewed as risky or unwise, the authors believe it is safe when proper the technique for calyceal access is achieved in select patients who satisfy all criteria for an ambulatory procedure. ${ }^{8,9}$ Further investigation in a larger population size is certainly required.
Competing interests: Andrea Kokorovic, Dr. Wilson and Dr. Beiko declare no competing financial or personal interests.

This paper has been peer-reviewed.

\section{References}

1. De Cogain MR, Krambeck AE. Advances in tubeless percutaneous nephrolithotomy and patient selection: An update. Curr Urol Rep 2013;14:130-7. http://dx.doi.org/10.1007/s1 1934-013-0310-4

2. Fernstrom I, Johannson B. Percutaneous pyelolithotomy: A new extraction technique. Scand J Urol Nephrol 1976;10:257-9.

3. Bellman GC, Davidoff R, Candela J, et al. Tubeless percutaneous renal surgery. J Urol 1997;157:1578-82. http://dx.doi.org/10.1016/S0022-5347(01)64799-2

4. Crook TJ, Lockyer CR, Keoghane SR, et al. A randomized controlled trial of nephrostomy placement versus tubeless percutaneous nephrolithotomy. J Urol 2008;180:612-4. http://dx.doi.org/10.1016/j. juro.2008.04.020

5. Garofalo $M$, Pultrone $C V$, Schiavina $R$, et al. Tubeless procedure reduces hospitalization and pain after percutaneous nephrolithotomy: Results of a multivariable analysis. Urolithiasis 2013;41:347-53. http:// dx.doi.org/10.1007/s00240-013-0565-8

6. Amer T, Ahmed K, Bultitude M, et al. Standard versus tubeless percutaneous nephrolithotomy: A systematic review. Urol Int 2012;88:373-82. http://dx.doi.org/10.1159/000336145

7. Wang J, Zhao C, Shang C, et al. Tubeless vs standard percutaneous nephrolithotomy: A meta-analysis. BJU Int 2012;109:918-24. http://dx.doi.org/10.1111/j.1464-410X.2011.10463.x

8. Shahrour W, Andonian S. Ambulatory percutaneous nephrolithotomy: Initial series. Urology 2010;76:128892. http://dx.doi.org/10.1016/i.urology.2010.08.001

9. Beiko D, Lee L. Outpatient tubeless percutaneous nephrolithotomy: The initial case series. Can Urol Assoc J 2010;4:E86-90.

10. Khairy Salem H, Morsi HA, Omran A, et al. Tubeless percutaneous nephrolithotomy in children. J Pediatr Urol 2007;3:235-8. http://dx.doi.org/10.1016/i.jpurol.2006.06.011

11. Jou YC, Lin CT, Shen CH, et al. Tubeless percutaneous nephrolithotomy for geriatric patients. Urol Int 2009;82:346-9. http://dx.doi.org/10.1159/000209370

12. $Y_{\cup} A$, Shahrour W, Andonian S. Simultaneous bilateral tubeless percutaneous nephrolithotomy: A report of 2 cases and review of the literature. Can Urol Assoc J 2012;6:E162-6.

Correspondence: Dr. Darren Beiko, Associate Professor, Department of Urology, Queen's University, Kingston General Hospital, 76 Stuart St., Kingston, ON K7L 2V7; fax: 613-545-1970; beiko@queensu.ca 